\title{
Results of the Patent Ductus Arteriosus Closure Using the Video-Assisted Thoracoscopic Surgery
}

\author{
Jamil Esfahanizadeh, ${ }^{1}$ Kambiz Alizadeh,,${ }^{2}$ Masoomeh Tabari, ${ }^{3}$ Azra Izanloo, ${ }^{4}$ Khadijeh Yaghobi, ${ }^{5}$ and
}

\author{
Mona Najaf Najafi ${ }^{6}$ \\ ${ }^{1}$ Associate Professor of Cardiac Surgery, Faculty of Medicine, Mashhad University of Medical Sciences, Mashhad, Iran \\ ${ }^{2}$ Assistant Professor of Cardiac Surgery, Department of Cardiac Surgery, Faculty of Medicine, Mashhad University of Medical Sciences, Mashhad, Iran \\ ${ }^{3}$ Associate Professor of Anesthesiology, Cardiac Anesthesia Research Center, Faculty of Medicine, Mashhad University of Medical Sciences, Mashhad, Iran \\ ${ }^{4}$ M.S.C in Medical Education, Cardiac Anesthesia Research Center, Mashhad University of Medical Sciences, Mashhad, Iran \\ ${ }^{5}$ Medical Student, Mashhad University of Medical Sciences, Mashhad, Iran \\ ${ }^{6}$ Assistant Professor of Community Medicine, Faculty of Medicine, Mashhad University of Medical Sciences, Mashhad, Iran \\ "Corresponding author: Kambiz Alizadeh, Assistant Professor of Cardiac Surgery, Department of Cardiac Surgery, Faculty of Medicine, Mashhad University of Medical Sciences, \\ Mashhad, Iran. Fax: +98-5138417402, E-mail: alizadehk@mums.ac.ir
}

Received 2016 December 03; Revised 2017 January 07; Accepted 2017 January 29.

\begin{abstract}
Background: Video-assisted thoracoscopic surgery (VATS) has been shown to be a safe and effective method for the patent ductus arteriosus (PDA) closure. This study aimed at evaluating the patients with PDA who underwent the VATS closure.

Methods: In this retrospective descriptive study, the medical records of 129 patients with the mean age of 35.44 months who underwent VATS were assessed during 2003 - 2016. Demographic data, mortality rate and surgery events were also recorded and reported. Results: The results showed that $9.3 \%$ of the patients had conversion to thoracotomy and less than $2.3 \%$ ( 3 cases) of the patients developed pulmonary complications: two chylothorax and one residual shunt. The mortality rate of these patients was zero. Conclusions: Results of this study showed that the PDA surgery using the VATS method could reduce the mortality rate, complications and side effects.
\end{abstract}

Keywords: PDA, Heart Surgery, Video-Assisted Thoracoscopic Surgery, VATS

\section{Background}

The patent ductus arteriosus (PDA) is the cause of more than $10 \%$ of congenital heart defects and it is more common among female infants than males $(1,2)$. The clinical symptoms depend on the volume of shunted blood from aorta to pulmonary artery bed, which is related to a diameter of duct and pulmonary vascular resistance.

As we know, untreated PDA can lead to an obstructive pulmonary disease, pulmonary hypertension, heart failure, and even increased risk of necrotizing enterocolis in premature infants (3). In premature infants, medical treatment is usually the first option and surgery is only considered when drug treatment is proved ineffective (4). Although the PDA ligation procedure is simple, three complications related to ligation including procedure-induced bleeding, unintended ligation of aortic, left pulmonary artery or its branches may occur (1). As a result, recently attempts have been made to find techniques that minimize these side effects.

One of these techniques is VATS. The present study aimed at evaluating patients with PDA who underwent VATS surgery during 2003 - 2016.

\section{Methods}

This is a retrospective descriptive study in which the medical records of 129 infants who underwent PDAligation during 2003 - 2016 in Ghaem Hospital, Mashhad, Iran were assessed. All operations were performed by the same surgeon and in all cases the necessity of the surgery for PDA ligation was confirmed by a pediatric cardiologist.

Information on 30-day mortality, lengths of intensive care unit (ICU) stay and total hospital stay, comorbidities and complications, including pulmonary complications, residual shunt and need for chest tube insertion was extracted from medical records and subjected to descriptive analysis.

All candidates for the VATS closure were referred to pediatric cardiologists. After arrival in operating room and standard monitoring, anesthesia was induced by sevoflurane or ketamine, fentanyl and atracurium. For maintenance, propofol infusion and fentanyl were used. Tracheal intubation was performed with an uncuffed endotracheal tube. An esophageal stethoscope was inserted after intubation.

All patients were positioned in right lateral decubitus. Three 5-mm incisions were created: anterior to scapula, 
posterior to scapula and tip of scapula. After dissection of PDA from aorta and pulmonary artery, and exposing of PDA, two clips were used for closure. Vanishing of PDA murmur was checked with an esophageal stethoscope.

The patients were extubated in the operating room or early in ICU.

We excluded all PDAs sized larger than $1 \mathrm{~cm}$ and patients that needed other operation(s) besides PDA closure.

\section{Results}

In this study, 129 patients underwent surgery during 2003 - 2016. Forty-six patients were males with the mean age of 35.44 months (age range, 2 to 144 months). More than $51 \%$ of the patients were under the age of 24 months. The average weight of the patients was $12.5 \pm 6.89 \mathrm{~kg}$ (range, 4 to $40 \mathrm{~kg}$ ). Six patients $(4.65 \%)$ had a Down syndrome and 13 patients (10.07\%) had other abnormalities like ventricular septal defect (VSD), patent foramen ovale (PFO), and floppy mitral valve. In 12 patients (9.3\%), thoracotomy was performed following VATS. It was due to technical problems like bleeding, and distorted anatomy. The average operation time was 0.52 hours. In 6 patients (4.65\%), a drain was inserted. Two subjects (1.55\%) had pulmonary complications and one showed residual shunt and was reoperated. The average length of stay in ICU and hospital was 2.89 hours and 1.78 days, respectively. There was no mortality in this period. The observed complications were as follows: there was one recurrent laryngeal nerve injury (with hoarseness); we also had two cases of chylothorax one of which is managed surgically. The 30-day mortality was zero.

The characteristics of the patients in the VATS group and the group that was eventually converted (thoracotomy) are shown in Table 1.

Table 1. Characteristics of Patients in the Video-Assisted Thoracoscopic Surgery and Convert Groups $^{\mathrm{a}}$

\begin{tabular}{lcc}
\hline \multirow{2}{*}{ Characteristics } & \multicolumn{2}{c}{ Frequency } \\
\cline { 2 - 3 } & VATS & Convert \\
\hline Recurrent laryngeal nerve injury & $1(0.77)$ & 0 \\
\hline Chylothorax & $2(1.55)$ & 0 \\
\hline Down Syndrome & $6(4.65)$ & 0 \\
Other associated anomalies & $13(10.07)$ & 0 \\
\hline Chest tube & $6(4.65)$ & $12(100)$ \\
Residual shunt & $1(0.77)$ & 0 \\
\hline $\begin{array}{l}\text { Abbreviation: VATS, Video-Assisted Thoracoscopic Surgery. } \\
\text { a Value are expressed as N. (\%). }\end{array}$ & \\
\hline
\end{tabular}

Some patients had disease other than PDA including 3 cases $(2.32 \%)$ of VSD, 3 cases $(2.32 \%)$ of PFO, 2 cases $(1.55 \%)$ of dilation AO, 2 cases (1.55\%) of dilation LA and LV and one case $(0.77 \%)$ of Floppy mitral. The PDA diameter is reported in Figure 1.

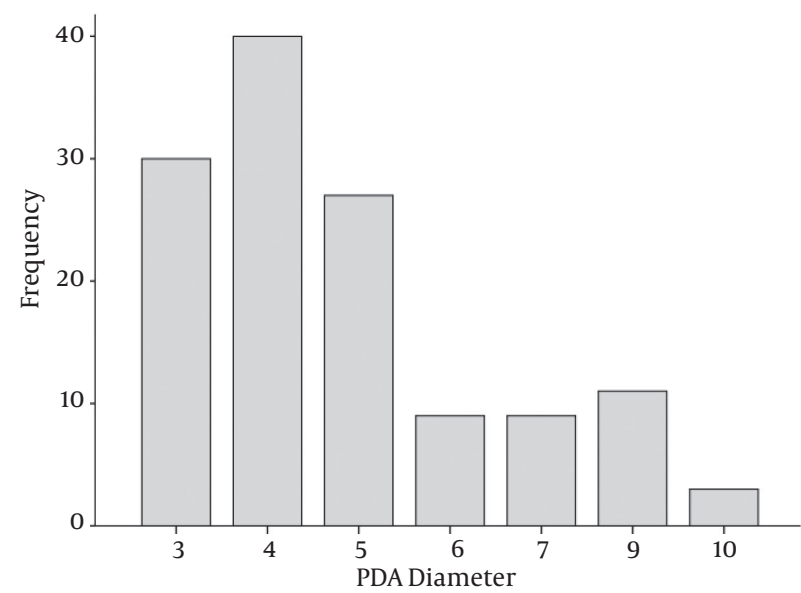

Figure 1. The Patent Ductus Arteriosus Diameter (mm)

\section{Discussion}

As we know, the PDA is one of the most common heart diseases in infants. Recently, there has been a tendency to perform this surgery by the VATS. Many studies have been conducted to examine the mortality rate of this method. For example, Satoko et al. (2016) reported a mortality rate of $9.9 \%$ and neurological disorder prevalence of $21.1 \%$ in this method (5), which depicts a declining trend compared to the previous studies (13\% and $44 \%$, respectively) (6). In our study, the 30-day mortality rate of patients was zero, though investigation with a larger sample size is required to confirm the results.

A meta-analysis by Weiz et al. in 2014 showed that the VATS surgery reduced mortality in patients, but it increased the prevalence of neurological disorders, respiratory problems and eye complications (7). The current study, however, did not report any of the complications observed in the mentioned study.

There were two important advantages in this method: first, cosmetic benefit for children that may be very important especially in psychological problems, and the second advantage, avoiding chest tube insertion in more than 95\% of the patients that allowed us to discharge them earlier from the hospital. It is important that the surgery was performed in early ages. Alipour et al. study has addressed it (8). 
The limitations of this study included its retrospective and single-center nature. However, the results suggest that the VATS surgery can be performed more conveniently with fewer side effects.

\subsection{Conclusion}

Results of this study showed that the PDA surgery by the VATS method can reduce the mortality rate, complications and side effects.

\section{References}

1. Banieghbal B. Physiological marker and surgical ligation of patent ductus arteriosus in neonates. Afr J Paediatr Surg. 2016;13(3):109-13. doi: 10.4103/0189-6725.187796. [PubMed: 27502877].

2. Clyman RI, Couto J, Murphy GM. Patent ductus arteriosus: are current neonatal treatment options better or worse than no treatment at all?. Semin Perinatol. 2012;36(2):123-9. doi: 10.1053/j.semperi.2011.09.022. [PubMed: 22414883].
3. Saldeno YP, Favareto V, Mirpuri J. Prolonged persistent patent ductus arteriosus: potential perdurable anomalies in premature infants.JPerinatol. 2012;32(12):953-8. doi: 10.1038/jp.2012.31. [PubMed: 22460543].

4. Sheridan BJ, Ward CJ, Anderson BW, Justo RN. Transcatheter closure of the patent ductus arteriosus: an intention to treat analysis. Heart Lung Circ. 2013;22(6):428-32. doi: 10.1016/j.hlc.2012.12.007. [PubMed: 23453524].

5. Ito S, Matsuda T, Usuda H, Watanabe S, Kitanishi R, Hanita T, et al. Surgical ligation for patent ductus arteriosus in extremely premature infants: Strategy to reduce their risk of neurodevelopmental impairment. Tohoku J Exp Med. 2016;240(1):7-13.

6. Madan JC, Kendrick D, Hagadorn JI, Frantz I3, National Institute of Child H, Human Development Neonatal Research N. Patent ductus arteriosus therapy: impact on neonatal and 18-month outcome. Pediatrics. 2009;123(2):674-81. doi: 10.1542/peds.2007-2781. [PubMed: 19171637]

7. Weisz DE, More K, McNamara PJ, Shah PS. PDA ligation and health outcomes: a meta-analysis. Pediatrics. 2014;133(4):e1024-46. doi: 10.1542/peds.2013-3431. [PubMed: 24639268].

8. Alipour MR, Mozaffari Shamsi M, Namayandeh SM, Pezeshkpour Z, Rezaeipour F, Sarebanhassanabadi M. The Effects of Oral Ibuprofen on Medicinal Closure of Patent Ductus Arteriosus in Full-Term Neonates in the Second Postnatal Week. Iran J Pediatr. 2016;26(4):e5807. doi: 10.5812/ijp.5807. [PubMed: 27729962]. 